\title{
Neonatal and Maternal Outcome of COVID-19 positive women in Sri Lanka: Secondary Analysis using National COVID-19 Positive Pregnant Women Surveillance
}

Malith Kumarasinghe ${ }^{1 *}$, Kaushalya Kasturiaratchi ${ }^{1}$, Hemali Jayakody ${ }^{1}$, Shakira $\operatorname{Irfaan}^{1}$, Wasana Samarasinghe $^{1}$, Harendra Dassanayake ${ }^{1}$, Sanjeeva Godakandage ${ }^{1}$, Chithramalee de Silva $^{1}$

*No 54/A, New Jayaweera Road, Ethul-Kotte, Sri Jayawardhanapura Kotte 10100. malith.kumarasinghe@yahoo.com

${ }^{1}$ Family Health Bureau, Ministry of Health, Colombo, Sri Lanka

\section{Objectives}

This study aims to describe the population level data on neonatal and maternal outcomes of COVID-19 positive pregnant women of Sri Lanka by secondary analysis using National COVID19 Positive Pregnant Women Surveillance.

\section{Design}

Secondary analysis of surveillance data from the National COVID-19 positive pregnant women surveillance, Sri Lanka. Data of all pregnant women whose maternal and neonatal outcomes were reported in National Surveillance from 1st March 2020 to 31st October 2021 were included in the study. Associated factors for maternal and neonatal outcomes, namely POA at delivery, mode of delivery, birthweight, immediate place of newborn care, congenital abnormalities, and condition of neonate at completion of one month were calculated using univariate and multivariate Odds ratios. 


\section{Results}

Maternal COVID-19 infection reported preterm birth rate of 11.9\%, LSCS rate of 54.5\%, low birthweight rate $16.5 \%$ and $8.3 \%$ of the newborns requiring intensive care. Neonatal mortality rate was 9 per 1000 live births. Pre-pregnancy overweight and obesity increased the risk of preterm delivery compared to pregnant women with normal BMI by 46.7\% (AOR=1.467, CI=1.111-1.938, $\mathrm{P}=0.007)$. In contrast, the risk of preterm delivery reduced by $82.4 \%$ (AOR=0.176, $\mathrm{CI}=0.097$ $0.317, \mathrm{p}<0.001)$ and presence of any type of congenital abnormalities in newborns by $72.4 \%$ among the COVID-19 positive women who required only inward treatment in comparison to women with severe COVID-19 infection requiring intensive care $(\mathrm{AOR}=0.276, \mathrm{CI}=0.112-0.683$, $\mathrm{p}=0.005)$.

\section{Conclusion}

Increased severity of maternal COVID-19 infection and pre-pregnancy overweight/ obesity were associated with many adverse pregnancy and neonatal outcomes. Therefore, close observation and aggressive management of COVID-19 among the pregnant women should be considered to reduce the risk of progressing to severe illness.

\section{Keywords: Maternal, COVID-19, Pregnancy outcomes, Neonatal Outcomes}




\section{INTRODUCTION}

In 2019, the novel coronavirus SARS-CoV-2 pandemic originated in Hubei Province of People's Republic of China. Within few months COVID-19 spread globally with majority of nations reporting cases. WHO Emergency Committee declared COVID-19 as a global health emergency on $30^{\text {th }}$ January 2020 and 11 th March 2020 as a pandemic.[1]

At the early stages of the pandemic, minimal knowledge existed on pregnancy and newborn outcomes of maternal COVID-19 infection unlike the understanding on disease severity and clinical profile. However, with the continuation of the pandemic, evidence on maternal and newborn outcomes in maternal COVID-19 gradually emerged. Many studies have highlighted the possibility of numerous adverse maternal and perinatal outcomes following COVID-19 infection.[2-6] Possible adverse maternal and perinatal outcomes include increased risk of preeclampsia, maternal death, disseminated intravascular coagulopathy, intrauterine fetal demise, intrauterine growth restriction, preterm delivery, spontaneous abortion, preterm birth and stillbirth. Further, systematic reviews further concluded that severe maternal COVID-19 infection significantly increase these adverse outcomes compared to mild to moderate infection.

An overview of systematic reviews reported that cesarean section is the preferred mode of delivery which ranged from $52.3 \%$ to $95.8 \%$.[7] Further, the study team reported the preterm delivery rate between $14.3 \%$ to $63.8 \%$. Stillbirth rate reported to be less than $2.5 \%$ and low birthweight between $5.3 \%$ to $47.4 \%$. NICU admission of the newborns of COVID-19 positive pregnant women ranged from $3.1 \%$ to $76.9 \%$ with neonatal mortality rate did not exceed 3\%. Despite the inclusion of 39 systematic reviews, the wide range of rates in pregnancy and neonatal outcomes highlight the nonagreement of the scientific community on the effect of maternal COVID-19 on pregnancy and neonatal outcomes.[7]

The literature revealed wide range in rates of pregnancy and neonatal outcomes in maternal COVID-19 infection, not only among the different studies but also in different geographical locations.[8-9] Systemic review by Dubey et al. revealed that deliveries by cesarean sections were higher among Chinese communities in comparison to American and European communities (91\% 
vs. $40 \%$ and $38 \%$ respectively). Similarly higher rates of adverse pregnancy outcomes were reported in Chinese studies compared to American and European studies (21\% vs 15\% and 19\% respectively). Further, the same systematic review showed reginal variation in preterm delivery. (American studies vs. Chinese and European studies: 12\% vs. 17\% and 19\% respectively).[8] These findings highlight the importance of local evidence for the management of pregnancy and neonatal outcomes of maternal COVID-19.

Though scientific information has emerged in developed countries on impact on COVID-19 maternal infection on pregnancy and newborn outcomes, the scarcity of information in developing countries, particularly in South Asia is a concern. In Sri Lanka, no literature is available on the pregnancy and newborn outcomes following maternal COVID-19 infection. Further, limited understanding prevails on factors which increase the risk of adverse pregnancy and neonatal outcomes in maternal COVID-19 infection. Therefore, this study was intended to answer 3 main questions related to pregnancy and newborn outcomes following maternal COVID-19 in Sri Lanka. Number one: To describe the basic sociodemographic, maternal, pregnancy and COVID19 disease related characteristics of COVID-19 positive pregnant women in Sri Lanka. Number two: To describe the factors significantly associated with the selected pregnancy outcomes among the COVID-19 positive pregnant women in Sri Lanka. Number three: To describe the factors significantly associated with the neonatal outcomes of COVID-19 positive pregnant women in Sri Lanka.

In this study, we aimed to describe the maternal, neonatal and COVID-19 disease related characteristics among the COVID-19 positive pregnant women in Sri Lanka using data from national COVID-19 positive pregnant women surveillance. In addition, the association of pregnancy and neonatal outcome variables were assessed with selected sociodemographic, maternal, pregnancy and COVID-19 disease related factors.

\section{METHODS}


Secondary analysis was conducted using the data available at National COVID-19 positive pregnant women surveillance in Sri Lanka.

\section{National COVID-19 positive pregnant women surveillance}

National COVID-19 positive pregnant women surveillance was initiated by the Ministry of Health to collect the information of COVID-19 positive pregnant women. The directive issued by the Director General of Health Services of Sri Lanka on 07th June 2021 instructed all health staff to register and follow up all COVID-19 positive pregnant women in Sri Lanka from 2020 onwards.[10] Therefore, data on positive women from the onset of the first case in Sri Lanka was gathered retrospectively and it is expected to continue until 2022. Information on COVID-19 positive pregnant women were reported by over 6000 field Public Health Midwife (PHM) in the country using a data extraction form. End of each month, the data was transferred to the electronic Reproductive Health Management Information System (eRHMIS) of Family Health Bureau. eRHMIS is the current electronic data management system of Reproductive, Maternal, New-born, Child, Adolescent, and Youth Health (RMNCAYH) programme in Sri Lanka based on the DHIS2 platform. The database included data of pregnant women who were tested positive either by RTPCR or by Rapid Antigen Test for SARS-CoV-2 virus.

Data extraction form comprised of four segments. First segment included socio-economic data, pregnancy details and other co-morbidities of all COVID-19 positive pregnant women. Segment two comprised of follow up information at the time of discharge. This included details on the severity of COVID-19 infection in women during the hospital stay and the pregnancy status at the time of discharge. PHM followed up the pregnant woman in the field and completed the third segment of the form at one month postpartum. It comprised information on both maternal and neonatal outcome. Fourth segment contained information of postnatal assessment of the infant at 6 months. The results of the 6 months assessment will be analysed and disseminated in future. 


\section{Study variables}

\section{Socio-demographic characteristics}

Age in years (grouped according to less than 35 years and 35 years or more), ethnicity, sector (urban, rural and estate according to the definition by the department of Census and Statistics) and monthly family (household) income according to the poverty line, median and mean household income.[11-12].

\section{Maternal characteristics}

Selected maternal characteristics include the pre-pregnancy BMI grouped as low, normal and overweight (and obesity), comorbidities, namely chronic DM, chronic hypertension, cardiovascular disease, chronic respiratory disease.

\section{Pregnancy and neonatal characteristics}

Pregnancy characteristics selected for the study include the presence of gestational DM, pregnancy induced HT, gravida, parity, number of living children, POA at delivery (preterm birth or not), mode of delivery (vaginal delivery or cesarian section). Neonatal characteristics include birthweight grouped according to WHO classification, place of immediate newborn care (postnatal ward or premature baby unit/ neonatal intensive care unit), initiation of breast feeding within one hour of birth, detection of any congenital abnormalities and neonate live or not at the end of neonatal period.[13]

\section{COVID-19 related measures}

COVID-19 vaccination status was categorized according to non-vaccinated or received at least single dose of any COVID-19 vaccination before 14 days of the diagnosis of COVID-19. Time of diagnosis of COVID-19 during the pregnancy was classified according to the trimester. Severity of the COVID-19 infection was categorized according to the place of the management (all COVID19 positive pregnant women mandate inward care as per Department of Health of Sri Lanka).[14] 


\section{Study methods and analysis}

Data extraction of all eligible COVID-19 positive pregnant women was carried out from the national surveillance (Eligibility- In the national surveillance, completed segments number one, two and three of the data extraction form by 31st October 2021) was extracted. Data was initially transferred to MS Excel software; they were coded and analysed using Statistical Package for Social Sciences (SPSS) 22 version. Rates were presented as proportions. Statistical significance was calculated based on a p value of less than 0.05 was considered as statistically significant. All variables were used to perform the multivariate analysis using logistic regression (enter method) irrespective of their significance in bivariate analysis to identify multivariate ORs and their Confidence Intervals. For monthly family (household) income, poverty line, median and mean household incomes were used for categorization.

\section{RESULTS}

\section{Description of the of the study population}

Total of 9905 pregnant women were identified as COVID-19 positive in National COVID-19 Positive Pregnant Women Surveillance by $31^{\text {st }}$ of October 2021. Majority of these cases were diagnosed in the $3^{\text {rd }}$ quarter of 2021 and therefore the delivery outcome of most of these women was not occurred at the time of the analysis. Thus, only in 2493 COVID-19 positive pregnant women, details of pregnancy and neonatal outcomes were available by 31st October 2021 in the surveillance. Out of these 2493 cases, 153 pregnant women reported miscarriages $\left(1^{\text {st }}\right.$ and $2^{\text {nd }}$ trimester) and was excluded from the analysis. Therefore, pregnancy and neonatal outcomes of 2340 COVID-19 positive pregnant women were included for the analysis.

Close to quarter of the COVID-19 positive pregnant women were below the poverty line (24.1\%).[12] Further, $80 \%$ of the pregnant women were below the median monthly household income of Sri Lanka.[12] Close to $18 \%$ of the pregnant women were 35 years or more (17.9\%). Pre-pregnancy overweight among the COVID-19 positive pregnant women stood at 35.7\%. Only

$31 \%$ reported receiving at least single dose of any type of COVID-19 vaccine. Incidence of 
Gestational DM was at 8\% whereas Pregnancy Induced Hypertension was reported as 3\% among the COVID-19 positive pregnant women (Table 1).

COVID-19 positive pregnant women showed preterm birth of $11.9 \%$ and LSCS rate at 54.5\%. Low birthweight rate was $16.5 \%$ with $8.3 \%$ of the newborns were admitted to either Premature Baby Unit or Neonatal Intensive Care Unit. Neonatal mortality rate was reported at 9 per 1000 live births among the COVID-19 positive pregnant women (Table 2).

\section{Table 1: Socio-demographic and maternal characteristics of COVID-19 positive pregnant women of Sri Lanka}

\begin{tabular}{|c|c|c|c|}
\hline \multicolumn{2}{|l|}{ Socio-demographic Variables } & \multicolumn{2}{|c|}{ No $(\%)$} \\
\hline & Less than 35 years & 1922 & $(82.1 \%)$ \\
\hline Age & 35 years or more & 418 & $(17.9 \%)$ \\
\hline \multirow{4}{*}{ Ethnicity } & Sinhala & 1458 & $(62.3 \%)$ \\
\hline & Tamil & 442 & $(18.9 \%)$ \\
\hline & Muslim & 439 & $(18.8 \%)$ \\
\hline & Burgher & 1 & $(0.0 \%)$ \\
\hline \multirow{3}{*}{ Sector } & Rural & 1739 & $(74.3 \%)$ \\
\hline & Urban & 515 & $(22.0 \%)$ \\
\hline & Estate & 86 & $(3.7 \%)$ \\
\hline \multirow{4}{*}{ Monthly Family Income } & Less than Rs $16,000 /=$ & 563 & $(24.1 \%)$ \\
\hline & Rs $16,001 /=$ to $\operatorname{Rs} 45,000 /=$ & 1309 & $(55.9 \%)$ \\
\hline & Rs $45,001 /=$ to Rs $65,000 /=$ & 323 & $(13.8 \%)$ \\
\hline & More than Rs $65,000 /=$ & 145 & $(6.2 \%)$ \\
\hline
\end{tabular}

\section{Maternal Variables}

\begin{tabular}{llrr}
\hline & Less than $18.5 \mathrm{~kg} / \mathrm{m}^{2}$ & 222 & $(9.7 \%)$ \\
\cline { 2 - 4 } Pre-pregnancy BMI & $18.5 \mathrm{~kg} / \mathrm{m}^{2}$ to $24.9 \mathrm{~kg} / \mathrm{m}^{2}$ & 1248 & $(54.6 \%)$ \\
\cline { 2 - 4 } & $25 \mathrm{~kg} / \mathrm{m}^{2}$ or more & 815 & $(35.7 \%)$ \\
\hline \multirow{3}{*}{ Chronic Diabetes mellitus } & No & 2285 & $(97.6 \%)$ \\
\cline { 2 - 5 } & Yes & 55 & $(2.4 \%)$ \\
\hline \multirow{2}{*}{ Chronic Hypertension } & No & 2323 & $(99.3 \%)$ \\
\cline { 2 - 5 } & Yes & 17 & $(0.7 \%)$ \\
\hline \multirow{2}{*}{ Cardiovascular Disease } & & & \\
\hline
\end{tabular}




\begin{tabular}{llrr}
\hline \multirow{2}{*}{ Chronic Respiratory Disease } & No & 2298 & $(98.2 \%)$ \\
\cline { 2 - 4 } & Yes & 42 & $(1.8 \%)$ \\
\hline \multirow{3}{*}{ Gestational Diabetes mellitus } & No & 2152 & $(92.0 \%)$ \\
\cline { 2 - 4 } & Yes & 188 & $(8.0 \%)$ \\
\hline \multirow{3}{*}{ Pregnancy induced Hypertension } & No & 2269 & $(97.0 \%)$ \\
\cline { 2 - 5 } & Yes & 71 & $(3.0 \%)$ \\
\hline \multirow{3}{*}{ Gravida } & One & 485 & $(20.7 \%)$ \\
\cline { 2 - 5 } & Two or more & 1855 & $(79.3 \%)$ \\
& & & \\
\hline \multirow{3}{*}{ Larity } & Zero & 1798 & $(76.8 \%)$ \\
\cline { 2 - 5 } & One or more & 820 & $(35.0 \%)$ \\
\hline \multirow{2}{*}{ COVID-19 vaccination } & Zero & 1520 & $(65.0 \%)$ \\
\cline { 2 - 5 } & One or more & & \\
\hline
\end{tabular}

Table 2: Pregnancy and neonatal characteristics of COVID-19 positive pregnant women of Sri Lanka

\begin{tabular}{llrr}
\hline Pregnancy Variables & & \multicolumn{2}{c}{ No (\%) } \\
\hline \multirow{3}{*}{ POA at Delivery } & 37 weeks or more & 2043 & $(88.1 \%)$ \\
\cline { 2 - 4 } & Less than 37 weeks & 276 & $(11.9 \%)$ \\
\hline & & & \\
\cline { 2 - 4 } Mode of Delivery & Normal Vaginal Delivery & 1028 & $(44.3 \%)$ \\
\cline { 2 - 4 } & Elective LSCS & 26 & $(1.1 \%)$ \\
\cline { 2 - 4 } & Emergency LSCS & 427 & $(36.1 \%)$ \\
\hline
\end{tabular}

\section{Neonatal Variables}

\begin{tabular}{llrr}
\hline & Extremely Low Birthweight & 8 & $(0.3 \%)$ \\
\cline { 2 - 4 } Birth Weight & Very Low Birthweight & 12 & $(0.5 \%)$ \\
\cline { 2 - 4 } & Low Birthweight & 363 & $(15.7 \%)$ \\
\cline { 2 - 4 } Place of Newborn Care following Birth & & 1934 & $(83.5 \%)$ \\
& Postnatal Ward only & 2135 & $(91.7 \%)$ \\
\cline { 2 - 4 } & Premature Baby Unit/ Neonatal & 194 & $(8.3 \%)$ \\
& Intensive Care Unit & & \\
\hline
\end{tabular}




\begin{tabular}{|c|c|c|c|}
\hline \multirow[b]{2}{*}{$\begin{array}{l}\text { Breast Feeding Initiated within One } \\
\text { Hour of Birth }\end{array}$} & Yes & 2267 & $(97.3 \%)$ \\
\hline & No & 62 & $(2.7 \%)$ \\
\hline \multirow{2}{*}{$\begin{array}{l}\text { Congenital Abnormalities detected } \\
\text { within Neonatal Period }\end{array}$} & No & 2265 & $(96.8 \%)$ \\
\hline & Yes & 75 & $(3.2 \%)$ \\
\hline \multirow{2}{*}{$\begin{array}{l}\text { Live Neonatal at the end of } 1^{\text {st }} 28 \text { days } \\
\text { following birth }\end{array}$} & Yes & 2319 & $(99.1 \%)$ \\
\hline & No & 21 & $(0.9 \%)$ \\
\hline
\end{tabular}

For OR calculations in Table 3 to 7 , above median to mean household income (Rs 45,001.00 to Rs 65,000.00) and above the mean household income groups were combined (More than 65,000.00). There was only single Burger COVID-19 positive pregnant woman. The said woman's mother tongue was Sinhala. Therefore, for OR calculation, the Burger pregnant woman was included to Sinhala ethnicity.(Burger population percentage is only $0.2 \%$ of Sri Lankan population according to census 2012).[11]

\section{Factors associated with preterm delivery of COVID-19 positive pregnant women}

Table three describes the selected sociodemographic, COVID-19, maternal, pregnancy and neonatal variables influence the time of delivery among COVID-19 positive pregnant women of Sri Lanka. Pre-pregnancy overweight and obesity increased the risk of preterm delivery among the COVID-19 positive women compared to normal BMI pregnant women by $46.7 \%$ (AOR=1.467, $\mathrm{CI}=1.111-1.938, \mathrm{P}=0.007)$. In contrast the risk of preterm delivery reduced by $82.4 \%$ among the COVID-19 positive women who required only inward treatment compared to the women needing ICU care or died due to COVID-19 (AOR=0.176, CI=0.097-0.317, $\mathrm{p}<0.001)$.

Table 3: Odds Ratios for factors associated with POA at delivery of COVID-19 positive pregnant women.
Univariate and multivariate analysis
\begin{tabular}{l|l|r|r|r|c|}
\hline $\begin{array}{l}\text { Outcome variable- POA at Delivery } \\
\text { 0 - 37 weeks or more } \\
\text { 1 - Less than 37 weeks }\end{array}$ & $\begin{array}{c}\text { 37 weeks } \\
\text { or more }\end{array}$ & $\begin{array}{c}\text { Less than } \\
\mathbf{3 7} \text { weeks }\end{array}$ & OR (Univariate) & OR (Multivariate) \\
\hline Age & 35 years or more & $350(84.7 \%)$ & $63(15.3 \%)$ & & \\
\cline { 2 - 6 } & Less than 35 years & $1693(88.8 \%)$ & $213(11.2 \%)$ & $0.699(0.516-0.947, \mathrm{p}=0.021)$ & $0.786(0.565-1.095, \mathrm{p}=0.154)$ \\
\hline
\end{tabular}


medRxiv preprint doi: https://doi.org/10.1101/2022.02.10.22270732; this version posted February 13, 2022. The copyright holder for this preprint (which was not certified by peer review) is the author/funder, who has granted medRxiv a license to display the preprint in perpetuity.

It is made available under a CC-BY-NC-ND 4.0 International license .

\begin{tabular}{|c|c|c|c|c|c|}
\hline \multirow[t]{3}{*}{ Ethnicity } & Tamil & $382(87.6 \%)$ & $54(12.4 \%)$ & & \\
\hline & Muslim & $383(88.2 \%)$ & $51(11.8 \%)$ & $0.942(0.626-1.417, \mathrm{p}=0.774)$ & $0.898(0.578-1.394, p=0.631)$ \\
\hline & Sinhala* & $1278(88.2 \%)$ & $171(11.8 \%)$ & $0.947(0.683-1.312, p=0.742)$ & $0.852(0.589-1.231, p=0.393)$ \\
\hline \multirow[t]{3}{*}{ Sector } & Urban & $448(87.8 \%)$ & $62(12.2 \%)$ & & \\
\hline & Estate & $78(90.7 \%)$ & $8(9.3 \%)$ & $0.741(0.342-1.608, p=0.448)$ & $0.733(0.318-1.691, p=0.467)$ \\
\hline & Rural & $1517(88.0 \%)$ & $206(12.0 \%)$ & $0.981(0.725-1.328, p=0.902)$ & $1.009(0.732-1.391, \mathrm{p}=0.956)$ \\
\hline \multirow{3}{*}{$\begin{array}{l}\text { Monthly } \\
\text { Family } \\
\text { Income }\end{array}$} & Rs $45,001 /=$ or more & $398(85.8 \%)$ & $66(14.2 \%)$ & & \\
\hline & Less than Rs $16,000 /=$ & $484(86.7 \%)$ & $74(13.3 \%)$ & $0.922(0.645-1.318, \mathrm{p}=0.656)$ & $0.960(0.645-1.431, p=0.843)$ \\
\hline & $\begin{array}{l}\text { Rs } 16,000 /=\text { to } \\
\operatorname{Rs} 45,000 /=\end{array}$ & $1161(89.5 \%)$ & $136(10.5 \%)$ & $0.706(0.515-0.968, p=0.031)$ & $0.744(0.533-1.038, p=0.082)$ \\
\hline \multirow{3}{*}{$\begin{array}{l}\text { Pre- } \\
\text { Pregnancy } \\
\text { BMI }\end{array}$} & $\begin{array}{l}18.5 \mathrm{~kg} / \mathrm{m}^{2} \text { to } \\
24.9 \mathrm{~kg} / \mathrm{m}^{2}\end{array}$ & $1112(89.7 \%)$ & $127(10.3 \%)$ & & \\
\hline & Less than $18.5 \mathrm{~kg} / \mathrm{m}^{2}$ & $202(91.4 \%)$ & $19(8.6 \%)$ & $0.824(0.497-1.364, p=0.451)$ & $0.845(0.505-1.412, p=0.519)$ \\
\hline & $25 \mathrm{~kg} / \mathrm{m}^{2}$ or more & $684(84.9 \%)$ & $122(15.1 \%)$ & $1.562(1.197-2.038, \mathrm{p}=0.001)$ & $1.467(1.111-1.938, \mathrm{p}=0.007)$ \\
\hline \multirow{2}{*}{$\begin{array}{l}\text { Presence of } \\
\text { Co- } \\
\text { morbidities }\end{array}$} & No & $1703(88.6 \%)$ & $220(11.4 \%)$ & & \\
\hline & Yes & $340(85.9 \%)$ & $56(14.1 \%)$ & $1.275(0.930-1.748, \mathrm{p}=0.131)$ & $1.084(0.775-1.515, \mathrm{p}=0.637)$ \\
\hline \multirow[t]{2}{*}{ Gravida } & One & $436(90.3 \%)$ & $47(9.7 \%)$ & & \\
\hline & Two or more & $1607(87.5 \%)$ & $229(12.5 \%)$ & $1.322(0.949-1.841, \mathrm{p}=0.099)$ & $1.176(0.497-2.783, p=0.712)$ \\
\hline \multirow[t]{2}{*}{ Parity } & One or more & $1558(87.5 \%)$ & $222(12.5 \%)$ & & \\
\hline & Zero & $485(90.0 \%)$ & $54(10.0 \%)$ & $0.781(0.571-1.070, p=0.124)$ & $1.012(0.417-2.456, \mathrm{p}=0.979)$ \\
\hline \multirow{2}{*}{$\begin{array}{l}\text { Living } \\
\text { Children }\end{array}$} & One or more & $1318(87.5 \%)$ & $189(12.5 \%)$ & & \\
\hline & Zero & $725(89.3 \%)$ & $87(10.7 \%)$ & $0.837(0.639-1.096, \mathrm{p}=0.195)$ & $1.074(0.707-1.630, p=0.739)$ \\
\hline \multirow{3}{*}{$\begin{array}{l}\text { POA at } \\
\text { Diagnosis }\end{array}$} & $3^{\text {rd }}$ Trimester & $1738(88.2 \%)$ & $233(11.8 \%)$ & & \\
\hline & $1^{\text {st }}$ Trimester & $32(80.0 \%)$ & $8(20.0 \%)$ & $1.865(0.849-4.095, \mathrm{p}=0.121)$ & $2.069(0.920-4.655, \mathrm{p}=0.079)$ \\
\hline & $2^{\text {nd }}$ Trimester & $273(88.6 \%)$ & $35(11.4 \%)$ & $0.956(0.656-1.395, \mathrm{p}=0.817)$ & $1.086(0.727-1.622, p=0.688)$ \\
\hline \multirow{2}{*}{$\begin{array}{l}\text { COVID-19 } \\
\text { Vaccination } \\
\text { Status }\end{array}$} & No & $1423(88.8 \%)$ & $179(11.2 \%)$ & & \\
\hline & At least Single Dose & $620(86.5 \%)$ & $97(13.5 \%)$ & $1.244(0.955-1.620, p=0.106)$ & $1.244(0.937-1.652, p=0.132)$ \\
\hline \multirow{2}{*}{$\begin{array}{l}\text { Severity of } \\
\text { COVID-19 } \\
\text { Infection }\end{array}$} & $\begin{array}{l}\text { ICU/ECMO/Ventilated/ } \\
\text { Death }^{\mathrm{a}}\end{array}$ & $32(59.3 \%)$ & $22(40.7 \%)$ & & \\
\hline & Inward care only & $2011(88.8 \%)$ & $254(11.2 \%)$ & $0.184(0.105-0.321, \mathrm{p}<0.001)$ & $0.176(0.097-0.317, \mathrm{p}<0.001)$ \\
\hline
\end{tabular}

Factors associated with delivery by cesarean section of COVID-19 positive pregnant women

Interestingly, lower income COVID-19 positive pregnant women (equal or less than monthly median family income) were less likely to undergo cesarean section (than vaginal delivery) compared to high income pregnant women (more than monthly median family income) $(\mathrm{AOR}=0.546, \mathrm{CI}=0.413-0.722, \mathrm{p}<0.001$ and $\mathrm{AOR}=0.578, \mathrm{CI}=0.459-0.729, \mathrm{p}<0.001)$. In addition, 
overweight or obese COVID-19 positive pregnant women demonstrated higher chance of undergoing cesarean section compared to normal BMI women with $23.3 \%$ increase (AOR=1.233, $\mathrm{CI}=1.021-1.490, \mathrm{p}=0.030$ ). In contrast, COVID-19 positive pregnant women with low prepregnancy BMI decreased the chances of undergoing cesarean section in comparison to women with normal pre-pregnancy BMI by 34.9\% (AOR=0.651, $\mathrm{CI}=0.482-0.878, \mathrm{p}=0.005)$. Presence of co-morbidities among the COVID-19 positive pregnant women increased the chance of undergoing cesarean section by 53.4\% (AOR=1.534, CI=1.210-1.945, p<0.001) (Table 4).

\section{Table 4: Odds Ratios for factors associated with mode of delivery of COVID-19 positive pregnant women. Univariate and multivariate analysis}

\begin{tabular}{|c|c|c|c|c|c|}
\hline \multicolumn{2}{|c|}{$\begin{array}{l}\text { Outcome variable- POA at Delivery } \\
\text { 0 - Vaginal (Normal or Assisted) } \\
1 \text { - LSCS (Elective or Emergency) }\end{array}$} & LSCS & $\begin{array}{c}\text { Vaginal } \\
\text { Delivery }\end{array}$ & OR (Univariate) & OR (Multivariate) \\
\hline \multirow[t]{2}{*}{ Age } & 35 years or more & $263(63.7 \%)$ & $150(36.3 \%)$ & & \\
\hline & Less than 35 years & $1002(52.6 \%)$ & $904(47.4 \%)$ & $0.632(0.507-0.788, \mathrm{p}<0.001)$ & $0.686(0.540-0.871, \mathrm{p}=0.002)$ \\
\hline \multirow[t]{3}{*}{ Ethnicity } & Tamil & $231(53.0 \%)$ & $205(47.0 \%)$ & & \\
\hline & Muslim & $223(51.4 \%)$ & $211(48.6 \%)$ & $0.938(0.719-1.224, \mathrm{p}=0.637)$ & $0.984(0.735-1.317, \mathrm{p}=0.912)$ \\
\hline & Sinhala* & $811(56.0 \%)$ & $638(44.0 \%)$ & $1.128(0.910-1.398, \mathrm{p}=0.271)$ & $1.016(0.795-1.298, \mathrm{p}=0.899)$ \\
\hline \multirow[t]{3}{*}{ Sector } & Urban & $271(53.1 \%)$ & $239(46.9 \%)$ & & \\
\hline & Estate & $41(47.7 \%)$ & $45(52.3 \%)$ & $0.804(0.509-1.270, \mathrm{p}=0.349)$ & $0.931(0.560-1.547, \mathrm{p}=0.781)$ \\
\hline & Rural & $953(55.3 \%)$ & $770(44.7 \%)$ & $1.092(0.895-1.331, \mathrm{p}=0.386)$ & $1.145(0.927-1.414, \mathrm{p}=0.209)$ \\
\hline \multirow{3}{*}{$\begin{array}{l}\text { Monthly } \\
\text { Family } \\
\text { Income }\end{array}$} & Rs $45,001 /=$ or more & $305(65.7 \%)$ & $159(34.3 \%)$ & & \\
\hline & Less than $\operatorname{Rs~} 16,000 /=$ & $282(50.5 \%)$ & $276(49.5 \%)$ & $0.533(0.413-0.686, \mathrm{p}<0.001)$ & $0.546(0.413-0.722, \mathrm{p}<0.001)$ \\
\hline & $\begin{array}{l}\text { Rs } 16,000 /=\text { to } \\
\operatorname{Rs} 45,000 /=\end{array}$ & $678(52.3 \%)$ & $619(47.7 \%)$ & $0.571(0.458-0.712, \mathrm{p}<0.001)$ & $0.578(0.459-0.729, \mathrm{p}<0.001)$ \\
\hline \multirow{3}{*}{$\begin{array}{l}\text { Pre- } \\
\text { Pregnancy } \\
\text { BMI }\end{array}$} & $\begin{array}{l}18.5 \mathrm{~kg} / \mathrm{m}^{2} \text { to } \\
24.9 \mathrm{~kg} / \mathrm{m}^{2}\end{array}$ & $660(53.3 \%)$ & $579(46.7 \%)$ & & \\
\hline & Less than $18.5 \mathrm{~kg} / \mathrm{m}^{2}$ & $89(40.3 \%)$ & $132(59.7 \%)$ & $0.591(0.442-0.791, \mathrm{p}<0.001)$ & $0.651(0.482-0.878, \mathrm{p}=0.005)$ \\
\hline & $25 \mathrm{~kg} / \mathrm{m}^{2}$ or more & $487(60.4 \%)$ & $319(39.6 \%)$ & $1.339(1.119-1.603, \mathrm{p}=0.001)$ & $1.233(1.021-1.490, \mathrm{p}=0.030)$ \\
\hline \multirow{2}{*}{$\begin{array}{l}\text { Presence of } \\
\text { Co- } \\
\text { morbidities }\end{array}$} & No & $1004(52.2 \%)$ & $919(47.8 \%)$ & & \\
\hline & Yes & $261(65.9 \%)$ & $135(34.1 \%)$ & $1.770(1.411-2.219, \mathrm{p}<0.001)$ & $1.534(1.210-1.945, \mathrm{p}<0.001)$ \\
\hline \multirow[t]{2}{*}{ Gravida } & One & $244(50.5 \%)$ & $239(49.5 \%)$ & & \\
\hline & Two or more & $1021(55.6 \%)$ & $815(44.4 \%)$ & $1.227(1.004-1.500, \mathrm{p}=0.046)$ & $1.032(0.580-1.837, \mathrm{p}=0.915)$ \\
\hline \multirow[t]{3}{*}{ Parity } & One or more & $990(55.6 \%)$ & $790(44.4 \%)$ & & \\
\hline & Zero & $275(51.0 \%)$ & $264(49.0 \%)$ & $0.831(0.685-1.008, \mathrm{p}=0.061)$ & $0.854(0.470-1.552, \mathrm{p}=0.606)$ \\
\hline & One or more & $837(55.5 \%)$ & $670(44.5 \%)$ & & \\
\hline
\end{tabular}




\begin{tabular}{|c|c|c|c|c|c|}
\hline $\begin{array}{l}\text { Living } \\
\text { Children }\end{array}$ & Zero & $428(52.7 \%)$ & $384(47.3 \%)$ & $0.892(0.752-1.059, \mathrm{p}=0.192)$ & $1.167(0.888-1.533, p=0.268)$ \\
\hline \multirow{3}{*}{$\begin{array}{l}\text { POA at } \\
\text { Diagnosis }\end{array}$} & $3^{\text {rd }}$ Trimester & $1105(56.1 \%)$ & $866(43.9 \%)$ & & \\
\hline & $1^{\text {st }}$ Trimester & $17(42.5 \%)$ & $23(57.5 \%)$ & $0.579(0.308-1.091, \mathrm{p}=0.091)$ & $0.537(0.279-1.034, p=0.063)$ \\
\hline & $2^{\text {nd }}$ Trimester & $143(46.4 \%)$ & $165(53.6 \%)$ & $0.679(0.534-0.864, \mathrm{p}=0.002)$ & $0.667(0.516-0.863, p=0.002)$ \\
\hline \multirow{2}{*}{$\begin{array}{l}\text { COVID-19 } \\
\text { Vaccination } \\
\text { Status }\end{array}$} & No & $857(53.5 \%)$ & $745(46.5 \%)$ & & \\
\hline & At least Single Dose & $408(56.9 \%)$ & $309(43.1 \%)$ & $1.148(0.961-1.371, \mathrm{p}=0.128)$ & $1.060(0.876-1.281, \mathrm{p}=0.551)$ \\
\hline \multirow{2}{*}{$\begin{array}{l}\text { Severity of } \\
\text { COVID-19 } \\
\text { Infection }\end{array}$} & $\begin{array}{l}\text { ICU/ECMO/Ventilated/ } \\
\text { Death }^{\mathrm{a}}\end{array}$ & $46(85.2 \%)$ & $8(14.8 \%)$ & & \\
\hline & Inward care only & $1219(53.8 \%)$ & $1046(46.2 \%)$ & $0.203(0.095-0.431, \mathrm{p}<0.001)$ & $0.208(0.092-0.470, \mathrm{p}<0.001)$ \\
\hline
\end{tabular}

\section{Factors associated with birthweight of the newborns of COVID-19 positive pregnant women}

Compared to COVID-19 positive pregnant women of 35 years or older, pregnant women aged less than 35 years were less likely to give birth to newborns with low birthweight by $44.6 \%$ $(\mathrm{AOR}=0.554, \mathrm{CI}=0.415-0.738, \mathrm{p}<0.001)$. In contrast, COVID-19 positive pregnant women belonging to estate sector displayed increased risk of giving birth to low birthweight newborns compared to unban pregnant women $(\mathrm{AOR}=2.413, \mathrm{CI}=1.344-4.334, \mathrm{p}=0.003)$. Similarly, COVID19 positive women with low pre-pregnancy BMI demonstrated increased risk compared to women with normal pre-pregnancy BMI by 74.6\% (AOR=1.746, $\mathrm{CI}=1.231-2.476, \mathrm{p}=0.002)$. In contrast the risk of low birthweight reduced by $69.9 \%$ among the COVID-19 positive women who required only inward treatment only compared to the women needing ICU care or death due to COVID-19 $(\mathrm{AOR}=0.301, \mathrm{CI}=0.165-0.551, \mathrm{p}<0.001)($ Table 5 $)$.

\begin{tabular}{|c|c|c|c|c|c|}
\hline \multicolumn{2}{|c|}{$\begin{array}{l}\text { Outcome variable- Birthweight } \\
0 \text { - } 2500 \mathrm{~g} \text { or more } \\
1 \text { - Less than } 2500 \mathrm{~g}\end{array}$} & $\begin{array}{l}2500 g \text { or } \\
\text { more }\end{array}$ & $\begin{array}{l}\text { Less than } \\
2500 \mathrm{~g}\end{array}$ & OR (Univariate) & OR (Multivariate) \\
\hline \multirow[t]{2}{*}{ Age } & 35 years or more & $317(77.1 \%)$ & $94(22.9 \%)$ & & \\
\hline & Less than 35 years & $1617(84.8 \%)$ & $289(15.2 \%)$ & $0.603(0.464-0.783, \mathrm{p}<0.001)$ & $0.554(0.415-0.738, \mathrm{p}<0.001)$ \\
\hline \multirow[t]{2}{*}{ Ethnicity } & Tamil & $345(79.1 \%)$ & $91(20.9 \%)$ & & \\
\hline & Muslim & $366(84.7 \%)$ & $66(15.3 \%)$ & $0.684(0.482-0.970, p=0.033)$ & $0.917(0.626-1.345, p=0.659)$ \\
\hline
\end{tabular}


medRxiv preprint doi: https://doi.org/10.1101/2022.02.10.22270732; this version posted February 13, 2022. The copyright holder for this preprint (which was not certified by peer review) is the author/funder, who has granted medRxiv a license to display the preprint in perpetuity.

It is made available under a CC-BY-NC-ND 4.0 International license .

\begin{tabular}{|c|c|c|c|c|c|}
\hline & Sinhala* & $1223(84.4 \%)$ & $226(15.6 \%)$ & $0.701(0.534-0.919, p=0.010)$ & $0.827(0.603-1.133, p=0.237)$ \\
\hline \multirow[t]{3}{*}{ Sector } & Urban & $442(86.7 \%)$ & $68(13.3 \%)$ & & \\
\hline & Estate & $60(69.8 \%)$ & $26(30.2 \%)$ & $2.817(1.664-4.767, \mathrm{p}<0.001)$ & $2.413(1.344-4.334, p=0.003)$ \\
\hline & Rural & $1432(83.2 \%)$ & $289(16.8 \%)$ & $1.312(0.987-1.744, p=0.062)$ & $1.277(0.949-1.718, p=0.107)$ \\
\hline \multirow{3}{*}{$\begin{array}{l}\text { Monthly } \\
\text { Family } \\
\text { Income }\end{array}$} & Rs $45,001 /=$ or more & $392(84.7 \%)$ & $71(15.3 \%)$ & & \\
\hline & Less than Rs $16,000 /=$ & $449(80.5 \%)$ & $109(19.5 \%)$ & $1.340(0.965-1.861, \mathrm{p}=0.080)$ & $1.176(0.817-1.693, p=0.382)$ \\
\hline & $\begin{array}{l}\text { Rs } 16,000 /=\text { to } \\
\text { Rs } 45,000 /=\end{array}$ & $1093(84.3 \%)$ & $203(15.7 \%)$ & $1.025(0.764-1.376, p=0.867)$ & $1.042(0.764-1.422, p=0.793)$ \\
\hline \multirow{3}{*}{$\begin{array}{l}\text { Pre- } \\
\text { Pregnancy } \\
\text { BMI }\end{array}$} & $\begin{array}{l}18.5 \mathrm{~kg} / \mathrm{m}^{2} \text { to } \\
24.9 \mathrm{~kg} / \mathrm{m}^{2}\end{array}$ & $1043(84.2 \%)$ & $196(15.8 \%)$ & & \\
\hline & Less than $18.5 \mathrm{~kg} / \mathrm{m}^{2}$ & $165(74.7 \%)$ & $56(25.3 \%)$ & $1.806(1.286-2.536, p=0.001)$ & $1.746(1.231-2.476, p=0.002)$ \\
\hline & $25 \mathrm{~kg} / \mathrm{m}^{2}$ or more & $683(85.0 \%)$ & $121(15.0 \%)$ & $0.943(0.737-1.206, p=0.639)$ & $0.912(0.705-1.179, p=0.482)$ \\
\hline \multirow{2}{*}{$\begin{array}{l}\text { Presence of } \\
\text { Co- } \\
\text { morbidities }\end{array}$} & No & $1608(83.7 \%)$ & $313(16.3 \%)$ & & \\
\hline & Yes & $326(82.3 \%)$ & $70(17.7 \%)$ & $1.103(0.829-1.467, \mathrm{p}=0.500)$ & $1.093(0.806-1.482, p=0.565)$ \\
\hline \multirow[t]{2}{*}{ Gravida } & One & $397(82.2 \%)$ & $86(17.8 \%)$ & & \\
\hline & Two or more & $1537(83.8 \%)$ & $297(16.2 \%)$ & $0.892(0.685-1.162, p=0.397)$ & $0.868(0.405-1.861, p=0.717)$ \\
\hline \multirow[t]{2}{*}{ Parity } & One or more & $1490(83.8 \%)$ & $288(16.2 \%)$ & & \\
\hline & Zero & $444(82.4 \%)$ & $95(17.6 \%)$ & $1.107(0.858-1.428, p=0.435)$ & $0.925(0.420-2.036, p=0.847)$ \\
\hline \multirow{2}{*}{$\begin{array}{l}\text { Living } \\
\text { Children }\end{array}$} & One or more & $1265(84.1 \%)$ & $240(15.9 \%)$ & & \\
\hline & Zero & $669(82.4 \%)$ & $143(17.6 \%)$ & $1.127(0.898-1.414, p=0.304)$ & $1.240(0.867-1.774, p=0.240)$ \\
\hline \multirow{3}{*}{$\begin{array}{l}\text { POA at } \\
\text { Diagnosis }\end{array}$} & $3^{\text {rd }}$ Trimester & $1643(83.4 \%)$ & $326(16.6 \%)$ & & \\
\hline & $1^{\text {st }}$ Trimester & $34(85.0 \%)$ & $6(15.0 \%)$ & $0.889(0.370-2.136, \mathrm{p}=0.793)$ & $0.970(0.397-2.372, p=0.947)$ \\
\hline & $2^{\text {nd }}$ Trimester & $257(83.4 \%)$ & $51(16.6 \%)$ & $1.000(0.724-1.382, \mathrm{p}=0.999)$ & $1.107(0.785-1.562, p=0.562)$ \\
\hline \multirow{2}{*}{$\begin{array}{l}\text { COVID-19 } \\
\text { Vaccination } \\
\text { Status } \\
\end{array}$} & No & $1643(83.4 \%)$ & $326(16.6 \%)$ & & \\
\hline & At least Single Dose & $588(82.2 \%)$ & $127(17.8 \%)$ & $1.136(0.899-1.435, p=0.286)$ & $1.113(0.866-1.429, \mathrm{p}=0.403)$ \\
\hline \multirow{2}{*}{$\begin{array}{l}\text { Severity of } \\
\text { COVID-19 } \\
\text { Infection }\end{array}$} & $\begin{array}{l}\text { ICU/ECMO/Ventilated/ } \\
\text { Death }^{\mathrm{a}}\end{array}$ & $34(63.0 \%)$ & $20(37.0 \%)$ & & \\
\hline & Inward care only & $1900(84.0 \%)$ & $363(16.0 \%)$ & $0.325(0.185-0.571, \mathrm{p}<0.001)$ & $0.301(0.165-0.551, \mathrm{p}<0.001)$ \\
\hline
\end{tabular}

\section{Factors associated with presence of congenital abnormalities of newborns of COVID-19 positive pregnant women}

Among the COVID-19 positive women who required only inward treatment, presence of any type of congenital abnormalities in newborn reduced by $72.4 \%$ in comparison to women with severe COVID-19 infection $(\mathrm{AOR}=0.276, \mathrm{CI}=0.112-0.683, \mathrm{p}=0.005)$ (Table 6). 


\section{Table 6: Odds Ratios for factors associated with the presence of congenital abnormalities of neonate of COVID-19 positive pregnant women. Univariate and multivariate analysis}

\begin{tabular}{|c|c|c|c|c|c|}
\hline \multicolumn{2}{|c|}{$\begin{array}{l}\text { Outcome variable- Congenital } \\
\text { abnormalities } \\
\text { 0 - Absent } \\
1 \text { - Present }\end{array}$} & Absent & Present & OR (Univariate) & OR (Multivariate) \\
\hline \multirow[t]{2}{*}{ Age } & 35 years or more & $399(95.5 \%)$ & $19(4.5 \%)$ & & \\
\hline & Less than 35 years & $1866(97.1 \%)$ & $56(2.9 \%)$ & $0.630(0.370-1.072, \mathrm{p}=0.089)$ & $0.656(0.371-1.161, \mathrm{p}=0.148)$ \\
\hline \multirow[t]{3}{*}{ Ethnicity } & Tamil & $431(97.5 \%)$ & $11(2.5 \%)$ & & \\
\hline & Muslim & $425(96.8 \%)$ & $14(3.2 \%)$ & $1.291(0.579-2.875, \mathrm{p}=0.532)$ & $1.399(0.604-3.242, \mathrm{p}=0.433)$ \\
\hline & Sinhala* & $1409(96.6 \%)$ & $50(3.4 \%)$ & $1.390(0.717-2.694, \mathrm{p}=0.329)$ & $1.341(0.649-2.771, \mathrm{p}=0.429)$ \\
\hline \multirow{3}{*}{$\begin{array}{l}\text { Monthly } \\
\text { Family } \\
\text { Income }\end{array}$} & Rs $45,001 /=$ or more & $455(97.2 \%)$ & $13(2.8 \%)$ & & \\
\hline & Less than Rs $16,000 /=$ & $547(97.2 \%)$ & $16(2.8 \%)$ & $1.024(0.487-2.151, \mathrm{p}=0.951)$ & $1.296(0.582-2.886, \mathrm{p}=0.526)$ \\
\hline & $\begin{array}{l}\text { Rs } 16,000 /=\text { to } \\
\operatorname{Rs} 45,000 /=\end{array}$ & $1263(96.5 \%)$ & $46(3.5 \%)$ & $1.275(0.682-2.381, \mathrm{p}=0.446)$ & $1.436(0.742-2.780, p=0.282)$ \\
\hline \multirow{3}{*}{$\begin{array}{l}\text { Pre- } \\
\text { Pregnancy } \\
\text { BMI }\end{array}$} & $\begin{array}{l}18.5 \mathrm{~kg} / \mathrm{m}^{2} \text { to } \\
24.9 \mathrm{~kg} / \mathrm{m}^{2}\end{array}$ & $1209(96.9 \%)$ & $39(3.1 \%)$ & & \\
\hline & Less than $18.5 \mathrm{~kg} / \mathrm{m}^{2}$ & $216(97.3 \%)$ & $6(2.7 \%)$ & $0.861(0.360-2.059, \mathrm{p}=0.737)$ & $0.982(0.405-2.383, \mathrm{p}=0.969)$ \\
\hline & $25 \mathrm{~kg} / \mathrm{m}^{2}$ or more & $788(96.7 \%)$ & $27(3.3 \%)$ & $1.062(0.645-1.749, \mathrm{p}=0.813)$ & $0.902(0.536-1.519, \mathrm{p}=0.698)$ \\
\hline \multirow{2}{*}{$\begin{array}{l}\text { Presence of } \\
\text { Co- } \\
\text { morbidities }\end{array}$} & No & $1883(97.3 \%)$ & $53(2.7 \%)$ & & \\
\hline & Yes & $382(94.6 \%)$ & $22(5.4 \%)$ & $2.046(1.230-3.404, p=0.006)$ & $1.813(1.046-3.143, \mathrm{p}=0.034)$ \\
\hline \multirow[t]{2}{*}{ Gravida } & One & $473(97.5 \%)$ & $12(2.5 \%)$ & & \\
\hline & Two or more & $1792(96.6 \%)$ & $63(3.4 \%)$ & $1.386(0.741-2.590, \mathrm{p}=0.307)$ & $0.662(0.082-5.324, \mathrm{p}=0.698)$ \\
\hline \multirow[t]{2}{*}{ Parity } & One or more & $1736(96.6 \%)$ & $62(3.4 \%)$ & & \\
\hline & Zero & $529(97.6 \%)$ & $13(2.4 \%)$ & $0.688(0.375-1.261, \mathrm{p}=0.226)$ & $0.528(0.064-4.373, \mathrm{p}=0.554)$ \\
\hline \multirow{2}{*}{$\begin{array}{l}\text { Living } \\
\text { Children }\end{array}$} & One or more & $1467(96.5 \%)$ & $53(3.5 \%)$ & & \\
\hline & Zero & $798(97.3 \%)$ & $22(2.7 \%)$ & $0.763(0.461-1.294, p=0.294)$ & $0.936(0.429-2.042, \mathrm{p}=0.868)$ \\
\hline \multirow{3}{*}{$\begin{array}{l}\text { POA at } \\
\text { Diagnosis }\end{array}$} & $3^{\text {rd }}$ Trimester & $1929(97.2 \%)$ & $56(2.8 \%)$ & & \\
\hline & $1^{\text {st }}$ Trimester & $37(92.5 \%)$ & $3(7.5 \%)$ & $2.793(0.836-9.331, \mathrm{p}=0.095)$ & $2.849(0.835-9.722, \mathrm{p}=0.095)$ \\
\hline & $2^{\text {nd }}$ Trimester & $299(94.9 \%)$ & $16(5.1 \%)$ & $1.843(1.044-3.255, \mathrm{p}=0.035)$ & $1.811(0.978-3.352, \mathrm{p}=0.059)$ \\
\hline \multirow{2}{*}{$\begin{array}{l}\text { COVID-19 } \\
\text { Vaccination } \\
\text { Status }\end{array}$} & No & $1561(96.7 \%)$ & $54(3.3 \%)$ & & \\
\hline & At least Single Dose & $704(97.1 \%)$ & $21(2.9 \%)$ & $0.862(0.517-1.439, \mathrm{p}=0.570)$ & $0.962(0.555-1.667, \mathrm{p}=0.890)$ \\
\hline \multirow{2}{*}{$\begin{array}{l}\text { Severity of } \\
\text { COVID-19 } \\
\text { Infection }\end{array}$} & $\begin{array}{l}\text { ICU/ECMO/Ventilated/ } \\
\text { Death }^{\mathrm{a}}\end{array}$ & $53(86.9 \%)$ & $8(13.1 \%)$ & & \\
\hline & Inward care only & $2212(97.1 \%)$ & $67(2.9 \%)$ & $0.201(0.092-0.439, \mathrm{p}<0.001)$ & $0.276(0.112-0.683, \mathrm{p}=0.005)$ \\
\hline
\end{tabular}


Factors associated with the place of immediate postnatal management of the newborn of COVID-19 positive pregnant women

Table seven demonstrates factors associated with the place of immediate management of the newborn of COVID-19 positive pregnant women of Sri Lanka. High pre-pregnancy BMI increased the risk of newborn admitting to PBU or NICU in comparison to pregnant women with normal BMI by $34.9 \%(\mathrm{AOR}=0.651, \mathrm{CI}=0.466-0.909, \mathrm{p}=0.012)$. In contrast, among the COVID-19 positive pregnant women only requiring inward care, the chances of the newborns only managed at postnatal ward increased significantly $(A O R=11.575, \mathrm{CI}=6.480-20.678, \mathrm{p}<0.001)($ Table 7$)$.

\section{Table 7: Odds Ratios for factors associated with the place of immediate postnatal management of the newborn of COVID-19 positive pregnant women. Univariate and multivariate analysis}

\begin{tabular}{|c|c|c|c|c|c|}
\hline \multicolumn{2}{|c|}{$\begin{array}{l}\text { Outcome variable- Place of } \\
\text { management of the newborn } \\
\text { 0 - Premature Baby Unit/ Neonatal } \\
\text { ICU (PBU/ NICU) } \\
1 \text { - Postnatal ward only }\end{array}$} & $\begin{array}{c}\text { PBU/ } \\
\text { NICU }\end{array}$ & $\begin{array}{l}\text { Postnatal } \\
\text { ward only }\end{array}$ & OR (Univariate) & OR (Multivariate) \\
\hline \multirow[t]{2}{*}{ Age } & 35 years or more & $43(10.4 \%)$ & $372(89.6 \%)$ & & \\
\hline & Less than 35 years & $151(7.9 \%)$ & $1763(92.1 \%)$ & $1.350(0.945-1.928, \mathrm{p}=0.100)$ & $1.357(0.909-2.028, \mathrm{p}=0.136)$ \\
\hline \multirow[t]{3}{*}{ Ethnicity } & Tamil & $38(8.7 \%)$ & $401(91.3 \%)$ & & \\
\hline & Muslim & $26(5.9 \%)$ & $411(94.1 \%)$ & $1.498(0.893-2.513, \mathrm{p}=0.126)$ & $1.418(0.806-2.496, p=0.225)$ \\
\hline & Sinhala* & $130(8.9 \%)$ & $1323(91.1 \%)$ & $0.964(0.661-1.408, \mathrm{p}=0.851)$ & $0.951(0.613-1.476, \mathrm{p}=0.824)$ \\
\hline \multirow[t]{3}{*}{ Sector } & Urban & $51(10.0 \%)$ & $461(90.0 \%)$ & & \\
\hline & Estate & $7(8.1 \%)$ & $79(91.9 \%)$ & $1.249(0.547-2.850, \mathrm{p}=0.598)$ & $1.262(0.509-3.129, \mathrm{p}=0.615)$ \\
\hline & Rural & $136(7.9 \%)$ & $1595(92.1 \%)$ & $1.297(0.925-1.819, \mathrm{p}=0.131)$ & $1.358(0.946-1.949, \mathrm{p}=0.098)$ \\
\hline \multirow{3}{*}{$\begin{array}{l}\text { Monthly } \\
\text { Family } \\
\text { Income }\end{array}$} & Rs $45,001 /=$ or more & $43(9.2 \%)$ & $422(90.8 \%)$ & & \\
\hline & Less than Rs $16,000 /=$ & $49(8.7 \%)$ & $513(91.3 \%)$ & $1.067(0.694-1.639, \mathrm{p}=0.768)$ & $0.802(0.493-1.305, \mathrm{p}=0.375)$ \\
\hline & $\begin{array}{l}\text { Rs } 16,000 /=\text { to } \\
\operatorname{Rs~} 45,000 /=\end{array}$ & $102(7.8 \%)$ & $1200(92.2 \%)$ & $1.199(0.825-1.741, \mathrm{p}=0.341)$ & $1.020(0.680-1.531, \mathrm{p}=0.923)$ \\
\hline \multirow{3}{*}{$\begin{array}{l}\text { Pre- } \\
\text { Pregnancy } \\
\text { BMI }\end{array}$} & $\begin{array}{l}18.5 \mathrm{~kg} / \mathrm{m}^{2} \text { to } \\
24.9 \mathrm{~kg} / \mathrm{m}^{2}\end{array}$ & $88(7.1 \%)$ & $1155(92.9 \%)$ & & \\
\hline & Less than $18.5 \mathrm{~kg} / \mathrm{m}^{2}$ & $15(6.8 \%)$ & $207(93.2 \%)$ & $1.051(0.596-1.854, \mathrm{p}=0.862)$ & $1.034(0.577-1.852, \mathrm{p}=0.911)$ \\
\hline & $25 \mathrm{~kg} / \mathrm{m}^{2}$ or more & $85(10.7 \%)$ & $725(89.5 \%)$ & $0.650(0.476-0.888, p=0.007)$ & $0.651(0.466-0.909, \mathrm{p}=0.012)$ \\
\hline \multirow{2}{*}{$\begin{array}{l}\text { Presence of } \\
\text { Co- } \\
\text { morbidities }\end{array}$} & No & $153(7.9 \%)$ & $1776(92.1 \%)$ & & \\
\hline & Yes & $41(10.3 \%)$ & $359(89.8 \%)$ & $0.754(0.525-1.084, \mathrm{p}=0.128)$ & $0.929(0.624-1.383, \mathrm{p}=0.717)$ \\
\hline \multirow[t]{2}{*}{ Gravida } & One & $41(8.5 \%)$ & $444(91.5 \%)$ & & \\
\hline & Two or more & $153(8.3 \%)$ & 1691(91.7\%) & $1.021(0.712-1.463, \mathrm{p}=0.912)$ & $0.683(0.289-1.619, p=0.387)$ \\
\hline Parity & One or more & $145(8.1 \%)$ & $1642(91.9 \%)$ & & \\
\hline
\end{tabular}




\begin{tabular}{|c|c|c|c|c|c|}
\hline & Zero & $49(9.0 \%)$ & $493(91.0 \%)$ & $0.888(0.633-1.247, p=0.494)$ & $0.785(0.321-1.916, \mathrm{p}=0.594)$ \\
\hline \multirow{2}{*}{$\begin{array}{l}\text { Living } \\
\text { Children }\end{array}$} & One or more & $116(7.7 \%)$ & $1397(92.3 \%)$ & & \\
\hline & Zero & $78(9.6 \%)$ & $738(90.4 \%)$ & $0.786(0.582-1.061, p=0.116)$ & $0.621(0.390-0.989, \mathrm{p}=0.045)$ \\
\hline \multirow{3}{*}{$\begin{array}{l}\text { POA at } \\
\text { Diagnosis }\end{array}$} & $3^{\text {rd }}$ Trimester & $165(8.3 \%)$ & $1812(91.7 \%)$ & & \\
\hline & $1^{\text {st }}$ Trimester & $3(7.5 \%)$ & $37(92.5 \%)$ & $1.123(0.343-3.682, p=0.848)$ & $1.115(0.323-3.854, \mathrm{p}=0.863)$ \\
\hline & $2^{\text {nd }}$ Trimester & $26(8.3 \%)$ & $286(91.7 \%)$ & $1.002(0.650-1.543, p=0.994)$ & $0.980(0.614-1.564, \mathrm{p}=0.932)$ \\
\hline \multirow{2}{*}{$\begin{array}{l}\text { COVID-19 } \\
\text { Vaccination } \\
\text { Status }\end{array}$} & No & $136(8.5 \%)$ & $1471(91.5 \%)$ & & \\
\hline & At least Single Dose & $58(8.0 \%)$ & $664(92.0 \%)$ & $1.058(0.768-1.459, \mathrm{p}=0.729)$ & $1.043(0.738-1.475, \mathrm{p}=0.812)$ \\
\hline \multirow{2}{*}{$\begin{array}{l}\text { Severity of } \\
\text { COVID-19 } \\
\text { Infection }\end{array}$} & $\begin{array}{l}\text { ICU/ECMO/Ventilated/ } \\
\text { Death }^{\mathrm{a}}\end{array}$ & $28(50.0 \%)$ & $28(50.0 \%)$ & & \\
\hline & Inward care only & $166(7.3 \%)$ & $2107(92.7 \%)$ & $12.693(7.344-21.937, \mathrm{p}<0.001)$ & $11.575(6.480-20.678, \mathrm{p}<0.001)$ \\
\hline
\end{tabular}

*Includes a single participant of Burger ethnicity (mother tongue was Sinhala)

aCU- intensive care unit; ECMO- extracorporeal membrane oxygenation

\section{DISCUSSION}

\section{Principal findings}

Analysis of pregnancy and neonatal characteristics of COVID-19 positive pregnant women of Sri Lanka revealed pre-pregnancy overweight rate of $35.7 \%$ with only $31 \%$ receiving at least single dose of any type of COVID-19 vaccine. Presence of gestational DM was at $8 \%$ and pregnancy induced hypertension at 3\% among the COVID-19 positive pregnant women. Maternal COVID19 infection reported preterm birth rate of $11.9 \%$ and LSCS rate of 54.5\%. Low birthweight rate was $16.5 \%$ with $8.3 \%$ of the newborns requiring intensive care among these women. Neonatal mortality rate was reported at 9 per 1000 live births among the COVID-19 positive pregnant women. Pre-pregnancy overweight and obesity increased the risk of preterm delivery among the COVID-19 positive women and increased the probability of undergoing cesarean section compared to normal BMI women. Similarly, presence of co-morbidities among these women increased the probability of undergoing cesarean section compared to women with no comorbidities. COVID-19 positive pregnant women belonging to estate sector displayed increased risk of giving birth to low birthweight newborns compared to unban pregnant women. The risk of low birthweight reduced by $69.9 \%$ among the COVID-19 positive women who required only inward treatment only compared to the women needing ICU care or death due to COVID-19. In addition, high pre-pregnancy BMI increased the risk of newborn admitting to PBU or NICU in comparison to pregnant women with normal BMI by $34.9 \%$. 


\section{National COVID-19 positive pregnant women surveillance}

The national COVID-19 positive pregnant women surveillance in Sri Lanka reported 9,441 cases of maternal COVID-19 was reported of which only 157 were reported in 2020. Majority of the maternal COVID-19 cases were reported from August to October of $2021(6,662)$.[15] Therefore, delivery outcome of majority of these pregnant women were not reported by 31 st of October which resulted in inclusion of only 2340 maternal COVID-19 cases out of 9,441. Number of maternal COVID-19 cases who were tested positive and not reported in the national surveillance is negligible due to several reasons. Centralized reporting of COVID-19 positive cases from all laboratories (both private and government) in Sri Lanka and the surveillance mechanism which forward these information to the relevant Medical Officer of Health areas for patient tracing and quarantine measures ensure that the field public health workers are updated on all COVID-19 positive cases including pregnant women. In addition, close to $95 \%$ of pregnant women of Sri Lanka have received domiciliary care by PHMs in 2019 which would have further reduced the possibility of missing positive maternal cases.[15]

\section{Socio-demographic and maternal characteristics}

As reported by Kumarasinghe et al. , percentage of pregnant women who were 35 years or older was $13.6 \%$ in Sri Lanka in 2020-2021 whereas present study revealed $17.9 \%$ of pregnant women in same age group highlighting the increase incidence of symptomatic COVID-19 infection with advancing age in Sri Lanka.[15] However, studies in America and Italy have suggested significant decrease in incidence of COVID-19 among the 35 years or above category in similar population based studies.[16-17] Onset and coverage of vaccination of pregnant women could be a factor in the observed difference. Though the countries like America and Italy initialed vaccinating pregnant women earlier than Sri Lanka, the coverage is an issue with large number of youth avoiding vaccination in America and Europe.[18-19]. In Sri Lanka, it is reported that around 80\% to 90\% of pregnant women in Sri Lanka was vaccinated against COVID-19 by September of 2021 being the first country in South Asia to commence vaccinating pregnant women ( Among the COVID19 positive women only $31 \%$ have received at least a single dose of any recommended vaccine before 14 days of the diagnosis.[20] 


\section{Preterm delivery}

Preterm delivery rate(less than 37 weeks) in the present study among COVID-19 positive pregnant women was $11.9 \%$. Published national preterm rates are not available for Sri Lanka. Many studies reported higher rates of preterm delivery among COVID-19 positive women compared to the present study. However, these studies were not population based studies and were confined to a single or multiple centers. These studies have only included pregnant women who were either tested positive on admission for the delivery or delivered during the hospital stay following admission to hospital with the diagnosis of COVID-19. Pre-term delivery rates ranged from $13.8 \%$ to $59.4 \% \cdot[8,21-25]$

Present study showed increased risk of preterm delivery among the pregnant women with severe COVID-19 infection. Conflicting evidence have emerged on whether the severity of the maternal COVID-19 infection influence the preterm delivery. A retrospective multicenter study in United States concluded that acute COVID-19 severity was not correlated with gestational age at delivery whereas separate cohort study in United States reported significant increase in preterm delivery among critical group compared to severe group.[22, 26] Our study reported preterm delivery rate of $40.7 \%$ among pregnant women needing ICU/ ECMO/ ventilated/ death whereas PierceWilliams reported preterm delivery rate of $27 \%$ among severe group and $88 \%$ among critical group.[22]

\section{Mode of Delivery}

Preferred mode of delivery of the COVID-19 positive pregnant women in Sri Lanka was cesarean section with $54.5 \%$ undergoing CS. The cesarean section rate of this study is among the one the highest among the published studies with large sample sizes. Not only among COVID-19 positive pregnant women, but national CS rate in Sri Lanka is also one of the highest in the world which might have contributed the increased CS rate of maternal COVID-19.[27-28] The CS rate among COVID-19 positive pregnant women ranged from 22.4\% in America to $94 \%$ in China. Some of these studies included less than 100 respondents.[8, 21-23, 26] 
Similar to the general population of pregnant women, pre-pregnancy overweight and obesity increased the chances of cesarean section among the maternal COVID-19 positive women in our study population. Significant increase in the CS rates were observed in United States cohort study with increase in severity of maternal COVID-19 infection in consistent with our study findings.[22]

\section{Birthweight of the newborn}

Present study revealed low birthweight of 16.5\%. Range of low birthweight reported by studies ranged from 5\% to $67 \%$. Many reasons could have contributed to this wide range. One could be the characteristics of the sample including the proportion of severe to critical women. Second, the inclusion criteria; Whether all women of past and present COVID-19 infection or only the women with active infection during the delivery was included. Third, the study setting, with some were institutional based whereas few were population based. Sample size also could have played a role in difference. However, it is important to compare the reported low birthweight rates with the national averages as many factors including sociodemographic factors play an important role in determining the low birthweight among COVID-19 positive pregnant women. Sri Lanka reported low birthweight of $16 \%$ in 2018.[28] Thus, only $0.5 \%$ increase in low birthweight was observed in the present study.[7-8, 25, 29-32]

Increase severity was associated with higher low birthweight rates as concluded by studies including the present one.[22] Our study revealed significantly higher rates of low birth weight among older pregnant women which is a known risk factor for low birthweight irrespective of the presence of COVID-19 among pregnant women. However, Dubey et al. in their meta-analysis failed to demonstrate statistical difference though the age categorization differed from the present study (35 or more vs. more than 30 years).[8, 33]

\section{Immediate Postnatal care and presence of congenital abnormalities}

As described under "birthweight of the newborn" many variations in study design, population, study setting and sample size have contributed to wide range of reporting rates of intensive 
postnatal care among maternal COVID-19 infection from 8.3\% (present study) to 76.9\%.[22-23, $30,32,34-35]$

Similar to present study, increased severity was associated with higher possibility of admission to intensive care for postnatal management of the newborn.[22] Further present study reported newborns of overweight/ obese pregnant women are at higher probability of admission to intensive care postnatally. Pre-pregnancy obesity in a known independent risk factor for neonatal intensive care admission. Therefore, the observed increased neonatal admission could be due to overweight/ obesity irrespective of the maternal COVID-19 status.[36]

Our study reported incidence of congenital abnormalities of any type and severity at $3.2 \%$. The national birth effect rate was less than $2 \%$. Therefore, there could be a possibility of increased incidence of congenital abnormalities among the newborns of COVID-19 positive pregnant women. At present, limited literature is available that provide clear direction on congenital abnormalities and COVID. Thus, further research is needed to have a clear picture.[37-38]

\section{CONCLUSION}

Increased severity of COVID-19 pregnant women was associated with many adverse pregnancy and neonatal outcomes including increased cesarean section rates, preterm delivery, low birthweight and congenital abnormalities compared to less severe infection. Similarly, prepregnancy overweight/ obesity could increase the risk of many negative pregnancy and neonatal outcomes compared to pregnant women with normal BMI. Thus, close observation and management of COVID-19 among the pregnant women should be considered to reduce the risk of progressing to severe illness. This could have an impact in reducing the adverse pregnancy and neonatal outcomes among maternal COVID-infection.

\section{Acknowledgements}

We acknowledge all the front-line public health staff, namely field Public Health Midwives, Public Health Nursing Sisters, Medical Officers of Health and regional health managers, namely Medical 
Officers- Maternal and Child Health for their contribution for the National COVID-19 Positive Pregnant Women Surveillance.

\section{Contributors}

HJ and KK developed the study protocol and overall workplan with JD and SG. MK was responsible for data extraction, cleaning, and analysis with the assistance of SI and WS. MK led the writing with all the authors contributed for writing and reviewing the final manuscript. CdS supervised the overall the project.

\section{Funding}

Study was self-funded.

\section{Disclaimer}

The views expressed are those of the authors and not necessarily those of the Family Health Bureau, Sri Lanka or Ministry of Health, Sri Lanka

\section{Competing interests}

All authors are key contributors of the National COVID-19 Positive Pregnant Women Surveillance of Sri Lanka

\section{Patient consent for publication}

Not applicable

\section{Ethics approval}


Ethical approval was obtained from Ethics Review Committee of Faculty of Medical Sciences, University of Sri Jayawardenepura (COVID 11/21).

\section{Data availability statement}

Data are available on reasonable request. Raw data without personal identifiers are available from corresponding author on reasonable request.

\section{REFERENCES}

1 World Health Organization. Coronavirus disease (COVID-19) pandemic, 2021. Available: https://www.who.int/emergencies/diseases/novel-coronavirus-2019 [Accessed 11th November 2021].

2 Epelboin S, Labrosse J, De Mouzon J, et al. Obstetrical outcomes and maternal morbidities associated with COVID-19 in pregnant women in France: A national retrospective cohort study. PLoS Med 2021;18:e1003857. https://doi.org/10.1371/journal.pmed.100385 [Accessed 19th November 2021].

3 Mehan A, Venkatesh A, Girish M. COVID-19 in pregnancy: Risk of adverse neonatal outcomes. J Med Virol. 2020;92:2295-2297. doi:10.1002/jmv.25959

4 Prochaska E, Jang M, Burd I. COVID-19 in pregnancy: Placental and neonatal involvement. Am J Reprod Immunol 2020;84:e13306. doi:10.1111/aji.13306

5 Barrero-Castillero A, Beam KS, Bernardini LB, et al. COVID-19: neonatal-perinatal perspectives. J Perinatol. 2021;41:940-951. doi:10.1038/s41372-020-00874-X 
6 Wei SQ, Bilodeau-Bertrand M, Liu S, et al. The impact of COVID-19 on pregnancy outcomes: a systematic review and meta-analysis. CMAJ. 2021;193:E540-E548. doi:10.1503/cmaj.202604

7 Papapanou, M, Papaioannou M, Petta A, et al. Maternal and Neonatal Characteristics and Outcomes of COVID-19 in Pregnancy: An Overview of Systematic Reviews. Int. J. Environ. Res. Public Health 2021;18:596. https://doi.org/10.3390/ijerph18020596 [Accessed 11th December 2021].

8 Dubey P, Reddy SY, Manuel S, et al. Maternal and neonatal characteristics and outcomes among COVID-19 infected women: An updated systematic review and meta-analysis. Eur J Obstet Gynecol Reprod Biol. 2020;252:490-501. doi:10.1016/j.ejogrb.2020.07.034

9 Salem D, Katranji F, Bakdash T. COVID-19 infection in pregnant women: Review of maternal and fetal outcomes. Int $J$ Gynaecol Obstet. 2021;152:291-298. doi:10.1002/ijgo.13533

10 Family Health Bureau. Information on COVID-19 positive pregnant mothers in the fieldFHB/EU/Gen/2021 dated $07^{\text {th }}$ June 2021 (2021).

11 Department of Census and Statistics. Census of Population and Housing 2012. Colombo: Department of Census and Statistics 2012. Available: http://www.statistics.gov.1k/pophousat/cph2011/pages/activities/reports/cph_2012_5per_r pt.pdf [Accessed 11th January 2022].

12 Central Bank of Sri Lanka. Economic and Social Statistics of Sri Lanka. Colombo: Central Bank of Sri Lanka, 2019. Available: https://www.cbsl.gov.lk/en/publications/otherpublications/statistical-publications/economic-and-social-statistics-of-sri-lanka [Accessed 11th January 2022]. 
13 World Health Organization. ICD-10 : international statistical classification of diseases and related health problems : tenth revision, 2nd ed. Geneva: World Health Organization 2004. Available: https://apps.who.int/iris/handle/10665/42980 [Accessed 21st January 2022].

14 Family Health Bureau. Guidelines for Management of Pregnant and Postpartum COVID19 patients- FHB/MCU/COVID/2021 dated 30 ${ }^{\text {th }}$ September 2021 (2021). Available: https://fhb.health.gov.lk/index.php/en/covid-19 [Accessed 15th January 2022].

15 Kumarasinghe M, Kasturiaratchi K, Jayakody H, et al. Secondary Analysis of Maternal Characteristics and Pregnancy Outcomes of COVID-19 Positive Pregnant Women in Sri Lanka Using National COVID-19 Positive Pregnant Women Surveillance. AJMAH. 2021;19:143-153. Available: https://doi.org/10.9734/ajmah/2021/v19i1230426. [Accessed $16^{\text {th }}$ January 2021].

16 D'Ambrosi F, Iurlaro E, Tassis B, et al. Sociodemographic characteristics of pregnant women tested positive for COVID-19 admitted to a referral center in Northern Italy during lockdown period. J Obstet Gynaecol Res. 2021;47:1751-1756. doi:10.1111/jog.14729

17 Ames JL, Ferrara A, Avalos LA, et al. COVID-19 prevalence, symptoms, and sociodemographic disparities in infection among insured pregnant women in Northern California. PLoS ONE. 2021;16:e0256891. https://doi.org/ 10.1371/journal.pone.0256891

18 UNICEF South Asia. COVID-19 and pregnancy: Staying safe, giving birth and getting vaccinated, 2021. Available: https://www.unicef.org/rosa/stories/covid-19-and-pregnancy [Accessed 18th January 2022].

19 Razzaghi H, Meghani M, Pingali C, et al. COVID-19 Vaccination Coverage Among Pregnant Women During Pregnancy - Eight Integrated Health Care Organizations, United States, December 14, 2020-May 8, 2021. Weekly. 2021;70:895-899. Available: https://www.cdc.gov/mmwr/volumes/70/wr/mm7024e2.htm [Accessed 20th January 2022]. 
20 Mudugamuwa I. Most pregnant women vaccinated now - FHB. Daily News, 2021. Available: https://www.dailynews.1k/2021/09/22/local/259957/most-pregnant-womenvaccinated-now-fhb [Accessed 2nd February 2022].

21 Martinez-Perez O, Rodriguez P, Hernandez M, et al. The association between SARS-CoV2 infection and preterm delivery: a prospective study with a multivariable analysis. $B M C$ pregnancy and childbirth. 2021;21:273. https://doi.org/10.1186/s12884-021-03742-4 [Accessed 2nd February 2022].

22 Pierce-Williams RAM, Burd J, Felder L, et al. Clinical course of severe and critical coronavirus disease 2019 in hospitalized pregnancies: a United States cohort study. Am J Obstet Gynecol. 2020;2(3):100134. doi:10.1016/j.ajogmf.2020.100134

23 Sentilhes L, De Marcillac F, Jouffrieau C, et al. Coronavirus disease 2019 in pregnancy was associated with maternal morbidity and preterm birth. Am J Obstet Gynecol. 2020;223(6):914.e1-914.e15. doi:10.1016/j.ajog.2020.06.022

24 Di Toro F, Gjoka M, Di Lorenzo G, et al. Impact of COVID-19 on maternal and neonatal outcomes: a systematic review and meta-analysis. Clin Microbiol Infect. 2021;27(1):3646. doi:10.1016/j.cmi.2020.10.007

25 Di Mascio D, Khalil A, Saccone G, et al. Outcome of coronavirus spectrum infections (SARS, MERS, COVID-19) during pregnancy: a systematic review and meta-analysis. Am J Obstet Gynecol. 2020;2(2):100107. doi:10.1016/j.ajogmf.2020.100107

26 Piekos SM, Roper RT, Hwang YM, et al. The effect of maternal SARS-CoV-2 infection timing on birth outcomes: a retrospective multicentre cohort study. The Lancet Digital 
Health. 2022;4(2):e95-e104 Available: https://doi.org/10.1016/S2589-7500(21)00250-8 [Accessed 3rd February 2022].

27 Gunasekera P, Wijesinghe P, Goonewardene I. The caesarean section rate is rising. Ceylon Med J. 2001;46(4):147-150. Available: http://doi.org/10.4038/cmj.v46i4.6466 [Accessed 3rd February 2022].

28 Family Health Bureau. Annual Report-2019. Colombo: Family Health Bureau, 2019. Available: https://fhb.health.gov.lk/index.php/en/resources/annual-report [Accessed 5th February 2022].

29 Ciapponi A, Bardach A, Comande D, et al. COVID-19 and pregnancy: An umbrella review of clinical presentation, vertical transmission, and maternal and perinatal outcomes. PLoS ONE. 2021;16(6):e0253974. https://doi. org/10.1371/journal.pone.0253974

30 Smith V, Seo D, Warty R, et al. Maternal and neonatal outcomes associated with COVID19 infection: A systematic review. PLoS One. 2020;15(6):e0234187. doi:10.1371/journal.pone.0234187

31 Trocado V, Silvestre-Machado J, Azevedo L, et al. Pregnancy and COVID-19: a systematic review of maternal, obstetric and neonatal outcomes. J Matern Fetal Neonatal Med. 2020;1-13. doi:10.1080/14767058.2020.1781809

32 Novoa RH, Quintana W, Llancarí P, et al. Maternal clinical characteristics and perinatal outcomes among pregnant women with coronavirus disease 2019. A systematic review. Travel Med Infect Dis. 2021;39:101919. doi:10.1016/j.tmaid.2020.101919

33 Aras RY. Is maternal age risk factor for low birth weight?. Arch Med Health Sci. 2022;1:33-7. Available: https://www.amhsjournal.org/text.asp?2013/1/1/33/113558 [Accessed 6th February 2022]. 
34 Lassi ZS, Ana A, Das JK, et al. A systematic review and meta-analysis of data on pregnant women with confirmed COVID-19: Clinical presentation, and pregnancy and perinatal outcomes based on COVID-19 severity. $J$ Glob Health. 2021;11:05018. doi:10.7189/jogh.11.05018

35 Diriba K, Awulachew E, Getu E. The effect of coronavirus infection (SARS-CoV-2, MERS-CoV, and SARS-CoV) during pregnancy and the possibility of vertical maternalfetal transmission: a systematic review and meta-analysis. Eur J Med Res. 2020;25(1):39. doi:10.1186/s40001-020-00439-w

36 Minsart AF, Buekens P, De Spiegelaere M, et al. Neonatal outcomes in obese mothers: a population-based analysis. BMC Pregnancy Childbirth. 2013;13:36. Available: https://doi.org/10.1186/1471-2393-13-36 [Accessed 5th February 2022].

37 World Health Organization. Birth Defects in Sri Lanka. World Health Organization, 2020. Available: https://www.who.int/srilanka/news/detail/03-03-2020-birth-defects-in-srilanka\#: :text=In\%20Sri\%20Lanka\%2C\%20around\%20360\%2C000,their\%20day\%20to \%20day\%20life [Accessed 8th February 2022].

38 Dube R, Kar SS. COVID-19 in pregnancy: the foetal perspective-a systematic review. BMJ Paediatrics Open. 2020;4:e000859. doi:10.1136/ bmjpo-2020-000859 